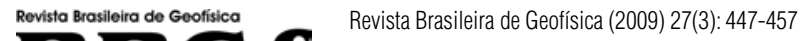 \\ RBGf: \\ Brazilian Jounal of Geophysics \\ www.scielo.br/rbg
}

\section{PLANEJAMENTO E CONTROLE DE QUALIDADE DE DADOS DE GRADIOMETRIA GRAVIMÉTRICA 3D-FTG}

\author{
Marco Antonio Braga ${ }^{1}$, Henry Galbiatti ${ }^{2}$, Dionísio Uendro Carlos $^{3}$, \\ Rodrigo Rocha Sousa ${ }^{4}$ e Tiago de Almeida ${ }^{5}$
}

Recebido em 25 maio, 2009 / Aceito em 18 setembro, 2009

Received on May 25, 2009 / Accepted on September 18, 2009

\begin{abstract}
This research job shows a detailed description of different steps of 3D-FTG (Full Tensor Gravity Gradiometry) planning and quality control (QC). The characteristics of 3D-FTG system, quality control during the flight and the result of the QC recorded through SNR (signal-to-noise ratio) analysis and the synthetical models of each gravity tensor component, will be presented. Iron oxide mineralization style is usually controlled by both crustal and local scale structures such as major shear, fault zones and lithological contacts. Air-FTG (Airborne Full Tensor Gravity Gradiometry) is nowadays the only existing airborne full tensor gradiometer technology can be used for both detailed and regional surveys. The system takes real time measurements of gravity gradient field in three orthogonal directions and at same time compensates for bias in the orientation and accelerations of the aircraft.
\end{abstract}

Keywords: signal-to-noise ratio, full tensor gravity gradiometry 3D-FTG, iron ore, Quadrilátero Ferrífero (MG).

RESUMO. 0 presente trabalho de pesquisa faz uma descrição detalhada das fases do planejamento e controle de qualidade dos dados de 3D-FTG (Full Tensor Gravity Gradiometry). Apresenta as características do sistema FTG, controle de qualidade dos dados durante o vôo e o resultado do controle de qualidade dos dados adquiridos através da análise da relação sinal ruído e dos modelos sintéticos de cada componente do tensor do gradiente de gravidade. Mineralizações de óxido de ferro são largamente controladas por estruturas crustais e locais, como zonas de cisalhamento, zonas de falha e contatos litológicos. Um controle cartográfico preciso destas estruturas é crítico para se encontrar concentrações consideráveis e econômicas destes tipos de depósitos. 0 Air-FTG (Airborne Full Tensor Gravity Gradiometry) é atualmente a única tecnologia aérea existente capaz de medir o tensor total gradiente de gravidade e pode ser usado tanto para levantamentos detalhados como regionais. 0 sistema aéreo FTG faz medidas em tempo real do gradiente do campo gravitacional em três direções ortogonais e ao mesmo tempo corrige a orientação e as acelerações da aeronave. A aquisição de dados com o sistema Air-FTG foi realizada no Quadrilátero Ferrífero, no estado de Minas Gerais, Brasil.

Palavras-chave: razão sinal ruído, gradiometria gravimétrica 3D-FTG, minério de ferro, Quadrilátero Ferrífero (MG).

Vale, Gerência de Exploração Mineral de Ferrosos, GAEMF, Av. de Ligação, 3580, Águas Claras, Centro de Tecnologia de Ferrosos, 34000-000 Nova Lima, MG, Brasil. Tel.: +55(31) 3215-4200 - E-mails: ${ }^{1}$ marco.antonio.braga@vale.com; ${ }^{2}$ henry.galbiatti@vale.com; ${ }^{3}$ dionisio.carlos@vale.com; ${ }^{4}$ rodrigo.rocha.sousa@vale.com; 5FTGeo-E-mail: ftgeologia@gmail.com 


\section{INTRODUÇÃo}

Recentemente, a Vale tem contratado levantamentos aerogeofísicos do tensor de gradiometria gravimétrica completo (3D-FTG) de alta resolução. Os espaçamentos das linhas de vôo são de aproximadamente 100 metros e a altura de vôo de aproximadamente 80 metros sendo esses vôos realizados no tipo drape, que consiste no acompanhamento, pelo piloto da aeronave, de uma superfície teórica calculada com base na performance de subida e descida de cada tipo de aeronave.

Como exemplo serão apresentados os dados de 3D-FTG adquiridos no Sinclinal Gandarela, região do Quadrilátero Ferrífero, estado de Minas Gerais. A região está inserida na Província São Francisco, situando-se no extremo sul da área ocupada pelo Cráton de nome homônimo (Almeida, 1977; Almeida \& Hassuy, 1984) e corresponde a um fragmento crustal poupado, em parte, da Orogênese Brasiliana, a qual abriga em seus domínios jazidas de ferro, ouro e outros recursos minerais.

\section{ÁREA DO LEVANTAMENTO GEOFísICO}

\section{Localização}

0 levantamento aerogeofísico de 3D-FTG foi realizado na parte sul do Sinclinal Gandarela região do Quadrilátero Ferrífero próximo aos municípios de Santa Bárbara e Barão de Cocais, estado de Minas Gerais, aproximadamente a $120 \mathrm{~km}$ da capital, Belo Horizonte. A Figura 1 apresenta a área de estudo.

0 Quadrilátero Ferrífero está inserido na Província São Francisco, situando-se no extremo sul da área ocupada pelo Cráton de mesmo nome e corresponde a um fragmento crustal poupado, em parte, da Orogênese Brasiliana, o qual abriga em seus domínios jazidas de ferro, ouro e outros recursos minerais.

\section{MATERIAIS E MÉTODOS}

\section{Planejamento da aquisição de dados}

A Figura 2 apresenta as linhas de vôo do levantamento de aerogradiometria gravimétrica. As mesmas foram orientadas na direção NE-SW. A altura do vôo foi de 80 metros, com espaçamento entre linhas de aproximadamente 100 metros, visando mapear os corpos de hematita, em um total de cinqüenta e quatro linhas levantadas que recobriram $927 \mathrm{~km}$ e aproximadamente $93 \mathrm{~km}^{2}$. Foi empregada uma aeronave Cessna Grand Caravan, modelo C208B, com velocidade de cruzeiro de $300 \mathrm{~km} / \mathrm{h}$ e velocidade em operação geofísica de $260 \mathrm{~km} / \mathrm{h}$.

Em áreas de topografia acidentada, como no Quadrilátero Ferrífero, deve-se programar o vôo drape de forma a garantir que as linhas de vôo sejam mais suaves, minimizando a quantidade de ruído durante a aquisição dos dados. Uma informação importante é que o sistema FTG mede o tensor total do gradiente da gravidade e as linhas de vôos não necessitam ser perpendiculares ao strike das camadas geológicas.

\section{Sistema FTG}

0 sistema FTG é instalado em uma cápsula aeronáutica, veja a Figura 3. A cápsula mede aproximadamente $1 \mathrm{~m}^{3}$ e possui massa de aproximadamente 227 quilogramas, com os GGl's (Gravity Gradiometer Instruments) instalados. 0 rack eletrônico tem aproximadamente as mesmas dimensões do sistema FTG e massa de 160 quilogramas. A cápsula aeronáutica fornece um ambiente de temperatura, pressão e umidade controlados para 0 FTG, durante toda a aquisiçã̃o de dados.

0 sistema Air-FTG ${ }^{\mathrm{TM}}$ é um instrumento altamente sensivel que requer um ambiente livre de ruídos e ao mesmo tempo estável, para obter o máximo de eficiência. Como essas duas condições não podem ser evitadas em sua totalidade, as vibrações e acelerações que contribuem de forma espúria devem ser administradas e/ou controladas. Além disso, o sistema FTG deve ser instalado próximo ao centro de gravidade da aeronave para reduzir os ruídos. A Figura 4 apresenta o equipamento montado numa aeronave Cessna Grand Caravan, modelo C-208B.

A aeronave Cessna Grand Caravan apresenta dimensões propícias para abrigar o sistema FTG e atualmente é a aeronave mais utilizada nesse tipo de aquisição de dados. Os ruídos e vibrações sofridas pela aeronave, tais como: rolagem, arfagem e derrapagem (roll, pitch e yaw, veja Fig. 5) que atuam no sistema ao longo de cada vôo, são monitoradas por dois acelerômetros externos e os dados são compensados destes ruídos espúrios.

Como 0 sinal do sistema Air-FTG ${ }^{\text {TM }}$ decai com 0 cubo da distância ao alvo, é importante voar 0 mais próximo possível ao terreno para detectar as pequenas amplitudes associadas aos corpos mineralizados. Para cumprir esse requisito é utilizada a técnica do vôo drape a uma altitude de 80 metros do solo. Esta é uma altura mínima de vôo que é considerada segura de acordo com os níveis de desempenho e segurança da aeronave.

Alguns critérios técnicos como a escolha do espaçamento entre as linhas de vôo, orientação das linhas e altura de vôo, são escolhas fundamentais para uma boa coleta de dados. Devem ainda, ser considerados: dimensões dos corpos mineralizados, orientação, topografia, contraste de densidade e profundidade dos alvos. 


\section{Gravação dos dados}

As medidas primárias das componentes do sistema FTG são gravadas inicialmente em um sistema de coordenadas interno que é referenciado aos três eixos dos GGl's. Estes dados são posteriormente transformados em um sistema de coordenadas com $x$ e $y$ no plano de superfície da Terra e $z$, perpendicular a esse plano, apontando para 0 interior da Terra.

Antes da aquisição um procedimento de auto-calibração é realizado com a aeronave em solo. Este procedimento gera uma tabela de fatores de calibração que serão utilizados no processamento dos dados para remover 0 efeito das variações de rolagem, arfagem e derrapagem que a aeronave sofrerá durante 0 vôo.

As três saídas do instrumento de gradiente gravimétrico gravam continuamente os níveis de ruído que são uma medida direta do desempenho operacional do sistema de aquisição. Os níveis de torque que ajustam os níveis de altitude da tabela dos GG/'s (Gravity Gradiometer Instruments) (em resposta as medidas inerciais de posição, velocidade e informações DGPS) também são gravados continuamente. As acelerações dos GGl's são mo- nitoradas para controle dos efeitos indesejáveis de turbulência da aeronave.

As linhas de vôo planejadas são mostradas no painel da aeronave, juntamente com 0 seu posicionamento, corrigido diferencialmente (DGPS), o que garantirá uma localização precisa da aeronave durante esta fase de aquisição.

As manobras de rolagem, arfagem e derrapagem da aeronave também foram continuamente gravadas para assegurar que as mesmas estivessem de acordo com as especificações de calibração do FTG.

Quando a aceleração vertical ultrapassa $60 \mathrm{mGal}$, a aquisição de dados é interrompida e as linhas que apresentam um nível de ruído acima desse valor são revoadas em condições meteorológicas mais favoráveis.

Para todos os dados gravados a equipe de campo se certifica que 0 sistema permaneça dentro de limites toleráveis, assegurando a consistência e alta qualidade dos resultados. Para garantir que os resultados estejam dentro das especificações, a equipe realiza análises diárias dos dados coletados, quais sejam:

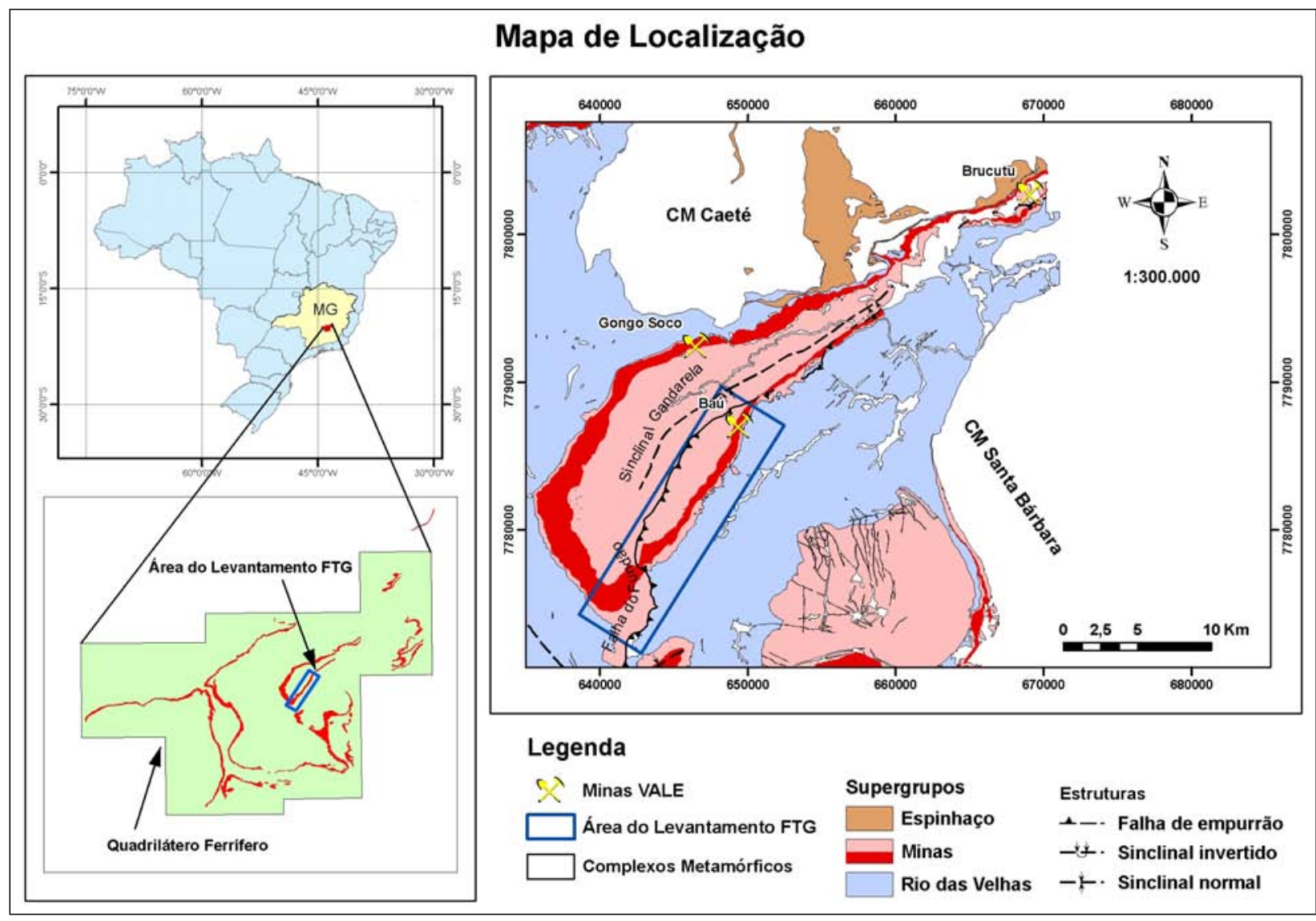

Figura 1 - Mapa da localização da área do levantamento geofísico 3D-FTG. 


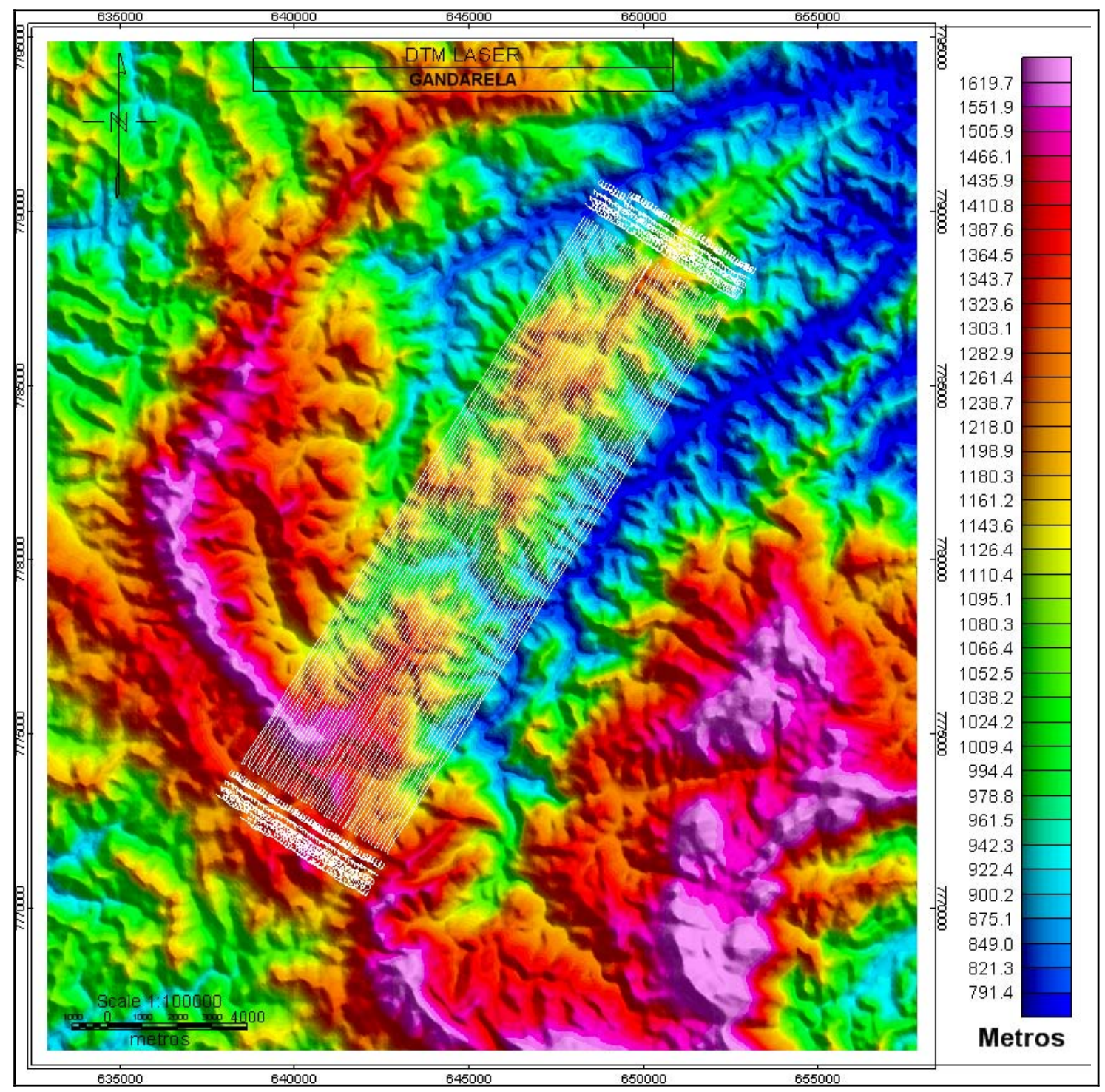

Figura 2 - Mapa do modelo digital do terreno, sobrepostas estão as linhas de vôo do levantamento aerogeofísico de aerogradiometria gravimétrica 3D-FTG. As linhas foram orientadas na direção NE-SW.

- Variáveis ambientais: ventos, fumaça, neblina;

- Posicionamento/navegação;

- Níveis de ruído dos GGl's, causados pelo vento e movimentos (roll, pitch e yaw);

- Aceleração vertical causada pelas correntes ascendentes.

Um registro detalhado da aquisição de dados e dos arquivos de controle de qualidade são gerados após cada vôo de produção, podendo ser consultado posteriormente.

\section{Controle de qualidade em vôo}

As acelerações medidas pelo equipamento durante a aquisição de dados são monitoradas durante 0 vôo e indicam 0 desempenho do sistema FTG. No visor do sistema FTG os operadores, a bordo da aeronave, inspecionam visualmente as somas lineares e os gradientes cruzados, posição e temperatura dos giroscópios, temperatura dos GG/'s e as acelerações norte, leste e vertical. Variações discordantes dos limites requeridos para 0 levantamento são constantemente verificadas e se as mesmas excedem os valores determinados a aquisição é interrompida automaticamente. 


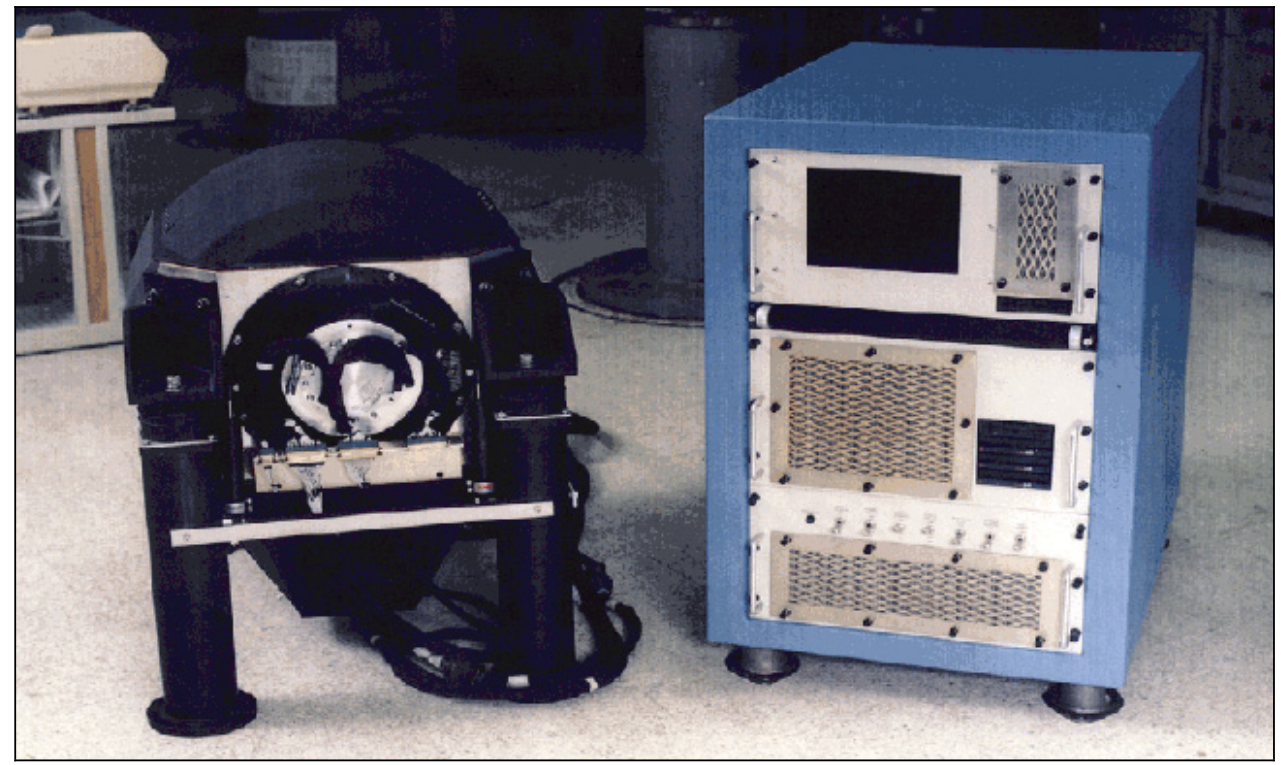

Figura 3 - Plataforma móvel do sistema FTG, a cápsula (à esquerda) mede aproximadamente $1 \mathrm{~m}^{3}$ tem massa de $227 \mathrm{~kg}$, com os GG/'s instalados. 0 rack eletrônico (à direita) tem dimensões aproximadas do sistema FTG e massa de aproximadamente $160 \mathrm{~kg}$.

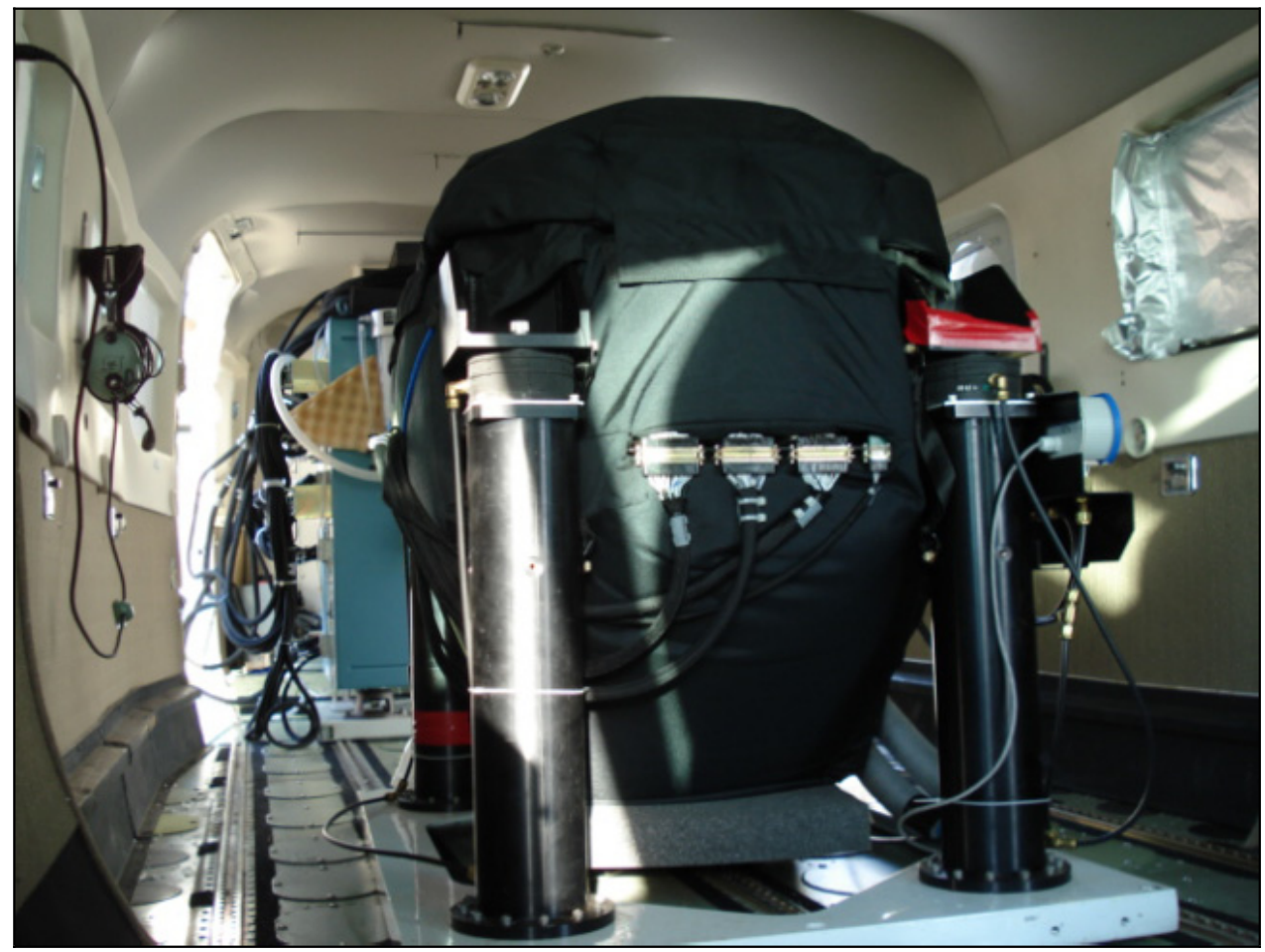

Figura 4 - Disposição dos sistemas dentro da aeronave. Em primeiro plano, a plataforma móvel do sistema FTG, ao fundo o rack. 


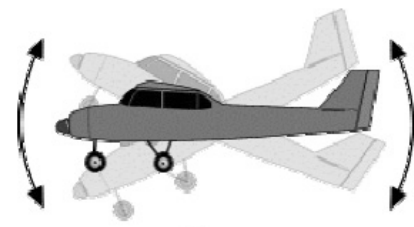

(a)

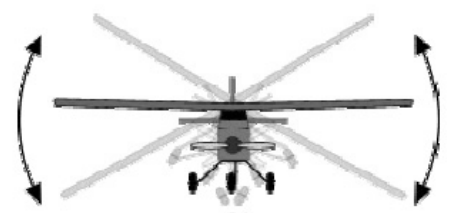

(b)

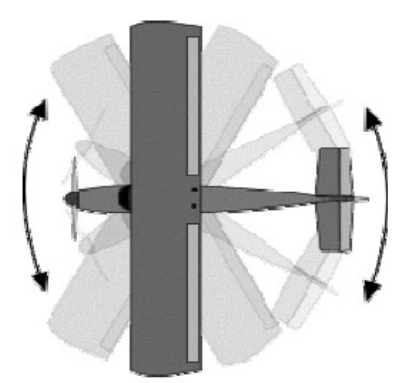

(c)

Figura 5 - Representação dos movimentos sofridos pela aeronave durante a aquisição de dados aerogeofísicos. (a) Pitch, (b) Rol/ e (c) Yaw. As acelerações externas são corrigidas por dois acelerômetros externos aos GGl's. Adaptado de Lasa (2007)

\section{Pré-processamento e conversão de dados de gradiometria gravimétrica}

As seguintes rotinas foram empregadas nos processamentos de dados de FTG:

a) High-Rate Post Mission Compensation (HRPMC) - Um filtro passa-baixa anti-aliasing é utilizado nessa fase antes da amostragem, para restringir os comprimentos de onda de alta freqüência. Essa fase de processamento consistiu na compensação das acelerações causadas por condições ambientais durante a aquisição do sistema FTG, usando registros simultâneos que monitoram o desempenho da plataforma GG/. Uma série de algoritmos, inerentes ao programa de aquisição, geram coeficientes para cada etapa do levantamento. Estes coeficientes são posteriormente aplicados aos dados para redução dos ruídos induzidos pelas acelerações espúrias.

b) Low-Rate Post Mission Compensation (LRPMC) - Esse software, também de propriedade da Bell Geospace, é baseado no filtro de Kalman, é um filtro passa-baixa e atua removendo os efeitos causados pelos ruídos que são inerentes as acelerações e velocidades. Esse processo tem por finalidade examinar os sinais inline (em linha) e crossline (cruzados) e gerar os valores das componentes Txx, Txy, Txz, Tyy, Tyz e Tzz. Este software realiza a etapa do processamento usando uma função de bias-spline no domínio do tempo, visando minimizar o drift ou erro nos dados de gradiente. Ao final deste processo os dados adquiridos a 1 kHz, são re-amostrados para 128 kHz e posteriormente para $1 \mathrm{~Hz}$ e transformados para as coordenadas reais em função de $x, y$ e $z$.

\section{Controle de qualidade}

Para um controle de qualidade mais apurado as linhas de vôo executadas no levantamento aerogeofísico 3D-FTG, são submetidos à Vale que mediante emprego de software próprio, realiza a avaliação da relação sinal ruído (SNR, signal-to-noise ratio) e uma vez que os dados apresentem um baixo valor na razão sinal ruído $(<30 \mathrm{~dB})$, as linhas são revoadas. Outro procedimento é 0 cálculo das componentes sintéticas das componentes do tensor gradiente de gravidade em função do modelo digital do terreno.

\section{Conceito da relação sinal ruído}

A razão sinal ruído é um conceito de engenharia elétrica, também utilizada em diversas aplicações que utilizam medidas de sinal em meio ruidoso. É definido como a razão entre potência de um sinal e a potência do ruído sobreposto ao sinal de interesse. A Equação 1 apresenta esse conceito.

$$
S N R=\frac{P_{\text {SINAL }}}{P_{\text {RUIDO }}}=\left(\frac{A_{\text {SINAL }}}{A_{\text {RUIDO }}}\right)^{2}
$$


onde:

$P=$ potência média;

$A=$ amplitude.

A relação sinal ruído é medida em decibel $(\mathrm{dB})$, veja a Equação 2 abaixo.

$$
S N R=10 \log _{10}\left(\frac{P_{\text {SINAL }}}{P_{\text {RUÍDO }}}\right)[d B]
$$

\section{Resultados obtidos na avaliação sinal ruído}

A Figura 6 apresenta as cinquenta e quatro linhas de vôo, num total de 927 quilômetros da região do Baú-Ganderala no Quadrilátero Ferrífero no estado de Minas Gerais.

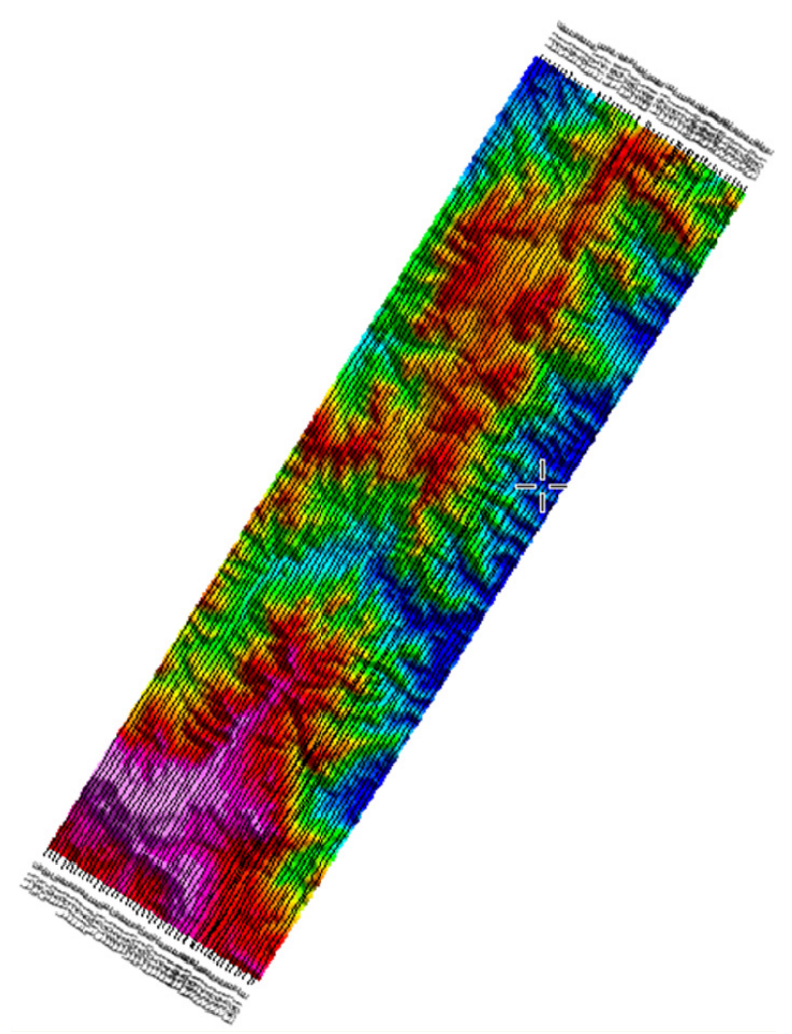

Figura 6 - Mapa do modelo digital do terreno da região do Baú-Gandarela. Sobrepostos à malha regular do terreno estão as linhas de vôo do levantamento aerogeofísico de 3D-FTG.

A avaliação da qualidade dos dados foi aplicada a componente $T_{z z}$ Ar-Livre de cada uma das linhas de vôo, pois esta representa diretamente a geologia da superfície e subsuperfície. Além disso, esta componente é influenciada pelos efeitos das acelerações espúrias externas sofridas pela aeronave, ruídos e vibrações causadas por fatores relacionados ao clima, temperatura e pressão.
A Figura 7 resume todas as linhas do levantamento aerogeofísico 3D-FTG da região do Baú-Gandarela.

0 resultado apresentado na Figura 7 mostra boa relação sinal ruído para as linhas de vôo, observa-se que das cinquenta e quatro linhas, cinquenta estão acima dos $30 \mathrm{~dB}$ e quatro linhas estão entre 28 e $30 \mathrm{~dB}$. Esses números representam dizer que nas piores condições, por exemplo as linhas L16, L18 e L34, apresentam mil vezes mais sinal do que ruído nos dados levantados na área do Gandarela, ao passo que nas melhores condições, por exemplo, linhas $L 10$ e L11, temos dez mil vezes mais sinal do que ruído nos dados.

Comparando-se as linhas L16, L18 e L34 com a linha L10 que apresenta maior valor da razão sinal ruído, estas seriam consideradas mais "ruidosas", porém, pelo fato de apresentarem um valor muito próximo do limite estabelecido de $30 \mathrm{~dB}$, foram consideradas de boa qualidade e não foram revoadas. Este fato é apresentado na Figura 8.

Para efeito comparativo 0 gráfico da Figura 9, mostra 0 resultado do controle de qualidade através da razão sinal ruído da componente $T_{z z}$ Free Air do levantamento de aerogradiometria gravimétrica 3D-FTG em outra área da Vale, onde foi possível detectar linhas de vôo muito ruidosas, com razão sinal ruído abaixo de $15 \mathrm{~dB}$.

A Figura 10 apresenta uma dessas linhas altamente ruidosa (L2161), situada entre linhas de ótima qualidade (linhas L2151 e L2171), para entendimento claro das linhas com baixa qualidade e que necessitam ser revoadas. A linha L2161 apresenta valor da razão sinal ruído de aproximadamente $12 \mathrm{~dB}$ e as linhas L2151 e L2171, respectivamente, valores de aproximadamente 37 e $28 \mathrm{~dB}$.

Apesar da linha L2161 ser bastante ruidosa, é possível notar a similaridade com as outras linhas imediatamente posterior e anterior a mesma, muito provavelmente causada pela resposta da componente $T_{z z}$ à geologia da área.

\section{Componentes sintéticas do tensor gradiente de gravidade}

Um modelo sintético para cada componente do tensor do gradiente de gravidade foi construído utilizando o modelo digital de terreno da região do Baú-Gandarela, a Equação 3, por exemplo apresenta 0 cálculo da componente $T_{z z}$. As demais componentes podem ser calculadas segundo Braga (2009). Foram utilizados a posição, altitude da aeronave e uma distribuição de densidades foi inserida na topografia para simular 0 efeito que essas massas causariam em cada uma das componentes do tensor. Este procedimento tem duas aplicações importantes: controle de 


\section{RAZÃO SINAL RUÍDO PARA CADA LINHA DE VÔO}

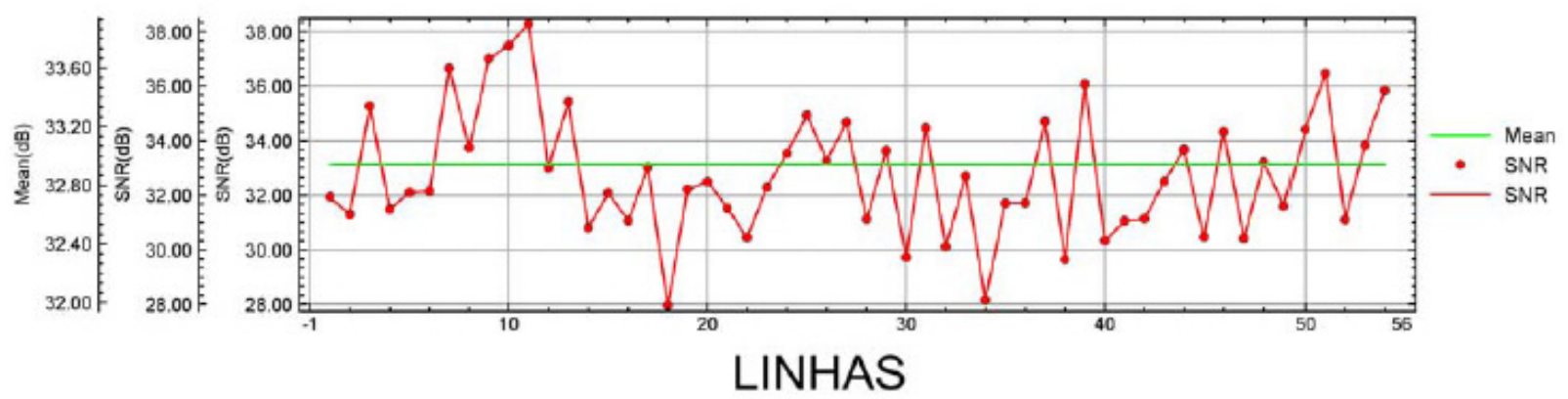

Figura 7 - Valores da razão sinal ruído da componente $T_{z z}$ Ar-Livre das linhas do levantamento aerogeofísico da região do Baú-Gandarela, Quadrilátero Ferrífero, Minas Gerais.

qualidade dos dados e a correção de terreno.

$$
T_{z z}(r)=\frac{\partial T_{z}}{\partial z}=\int_{M D T} \frac{\partial^{2}}{\partial z^{2}} G\left(r-r^{\prime}\right) \rho d^{3} r^{\prime}
$$

Após o cálculo das componentes sintéticas do tensor, como controle de qualidade, é possível fazer uma comparação entre as componentes calculadas e as componentes Ar-Livre adquiridas pelo sistema FTG, observe a Figura 11. É esperado que existam algumas semelhanças entre as componentes calculadas e medidas, devido ao fato dos dados de Ar-Livre sofrerem influência direta da topografia.

De posse do resultado da componente $T_{z z}$ sintética e da componente $T_{z z}$ Free Air da região do Baú-Gandarela, Quadrilátero Ferrífero, Minas Gerais, é possível fazer uma correlação entre elas, visando quantificar as similaridades, pois os dados do $T_{z z}$ Free Air sofrem influência direta da topografia e também da geologia da região investigada.

A Figura 12 mostra 0 resultado da correlação entre a componente $T_{z z}$ sintética e a componente $T_{z z}$ Free Air em todas as linhas de vôo na região do Baú-Gandarela, Quadrilátero Ferrífero, Minas Gerais. Como é possível perceber, a correlação é muito boa, a linha 10 ( 90\%) é a que apresenta maior correlação entre os dois dados e a linha $16(\sim 35 \%)$ a menor correlação. Se comparados esses dados com o resultado do SNR veremos que a linha 10, que apresenta maior correlação entre as componentes $T_{z z}$ tem um SNR de $37.03 \mathrm{~dB}$ e a linha 16 que tem uma correlação menor entre as componentes $T_{z z}$ apresenta um SNR de $31,07 \mathrm{~dB}$.

\section{CONCLUSÃO}

0 planejamento da aquisição de dados de aerogradiometria gravimétrica 3D-FTG deve levar em consideração a topografia da região, pois o sistema Air-FTG é muito sensível às acelerações verticais relacionadas à turbulência e às manobras roll, pitch e yaw da aeronave, embora essas sejam continuamente gravadas e posteriormente corrigidas. As linhas de vôo não precisam ser perpendiculares ao strike das camadas geológicas. 0 importante é que as mesmas não sofram variações grandes e/ou abruptas, em função da topografia, pois assim a quantidade de ruído inserida nos dados, durante a aquisição será menor.

0 controle de qualidade dos dados de aerogradiometria gravimétrica 3D-FTG, mostrados aqui através da razão sinal ruído se mostrou fundamental na etapa de controle de qualidade feita pela Vale, durante a aquisição de dados, garantindo assim a melhor qualidade possível para os dados coletados. A comparação entre as componentes sintéticas e as componentes Free Air do tensor também se mostrou uma ferramenta eficaz para o controle de qualidade uma vez que as componentes sintéticas são calculadas a partir do modelo digital de terreno da área e suas variações para com os dados Free Air não têm de ser grandes.

\section{AGRADECIMENTOS}

Os autores agradecem à Vale S.A., especialmente à Diretoria de Ferrosos pela oportunidade de publicação deste trabalho.

\section{REFERÊNCIAS}

ALMEIDA FFM. 1977. 0 Cráton do São Francisco. Revista Bras. Geoc., 7(4): 349-364.

ALMEIDA FFM \& HASSUY Y (Coords.). 1984. 0 Precambriano do Brasil. São Paulo. Edgard Blucher. $542 \mathrm{p}$.

BRAGA MA. 2009. Aplicação de Aerogradiometria Gravimétrica 3D-FTG na Prospecção de Minério de Ferro no Quadrilátero Ferrífero (MG) e Modelagem 2D e 3D para Estimativa de Massa do Depósito de Ferro de N1 em Carajás (PA); UFRJ, Tese de Doutorado. 271 p.

LASA. 2007. Levantamento Aéreo de Magnetometria, área "Cerra 9" Minas Gerais, volume 1. 


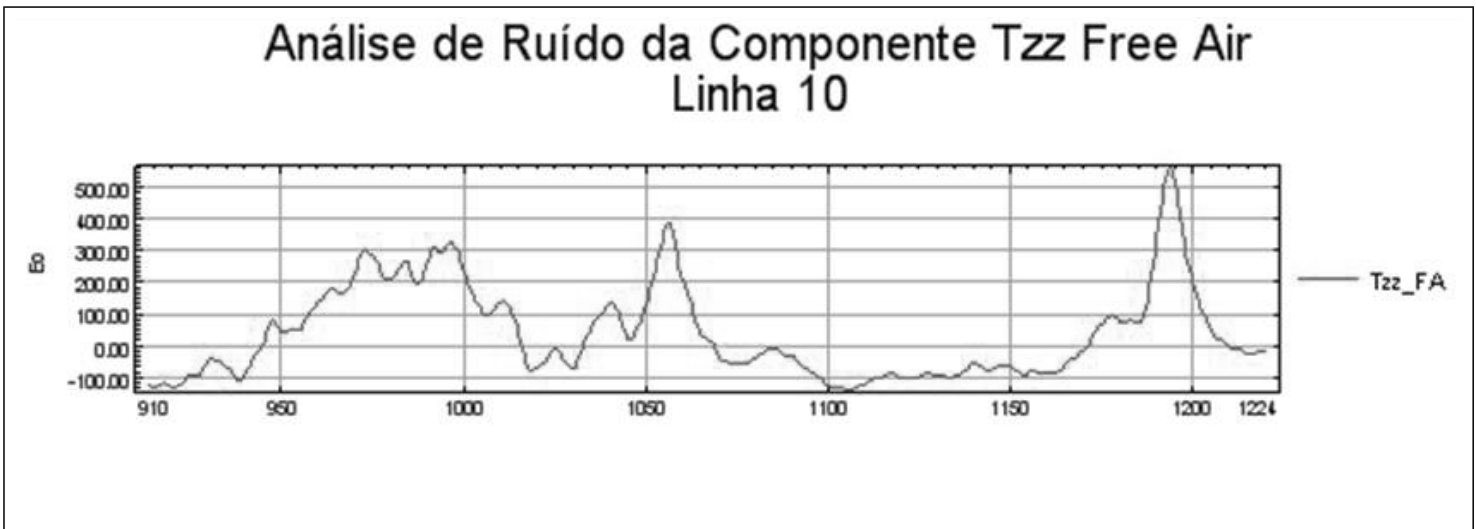

\section{Análise de Ruído da Componente Tzz Free Air Linha 16}
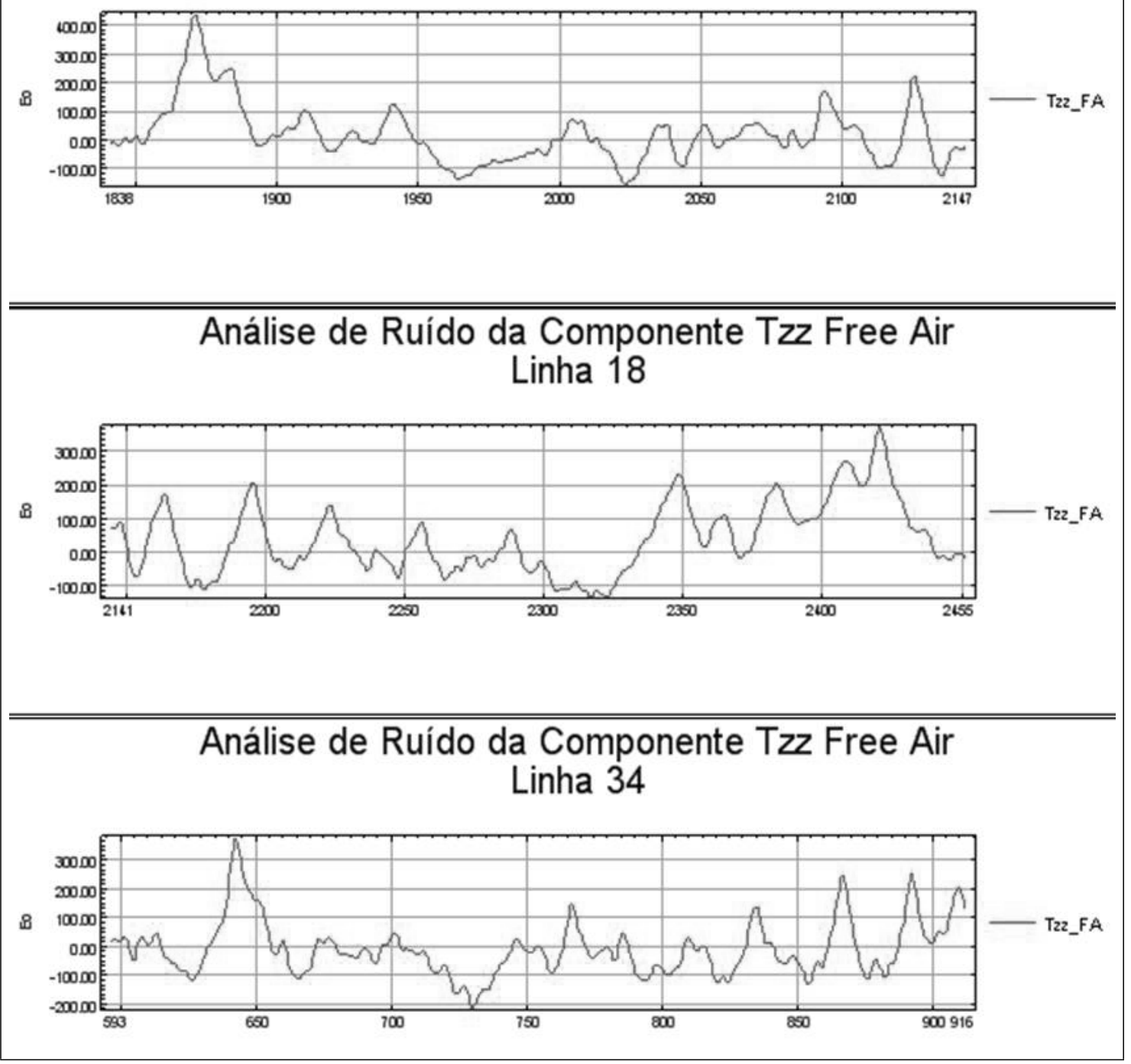

Figura 8 - Representação em perfil da componente $T_{z z}$ Free Air das linhas L10, L16, L18 e L34 do levantamento aerogeofísico da região do Baú-Gandarela. Note como as linhas L16, L18 e L34 apresentam sinais de alta freqüência (mais ruidosas) que a primeira linha (L10), mais suavizada. 
RAZÃO SINAL RUÍDO - CEBUS 10

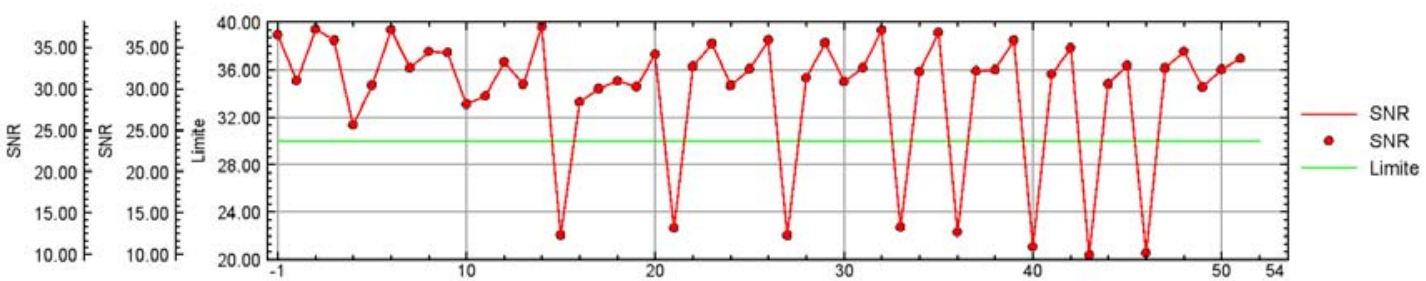

Figura 9 - Razão sinal ruído da componente $T_{z z}$ Free Air das 51 linhas de vôo do levantamento de aerogradiometria gravimétrica 3D-FTG em outra área (Cebus 10) da Vale. Um total de 43 linhas estão acima de $30 \mathrm{~dB}$ e 8 linhas de vôo que estão com a razão sinal ruído menor que $15 \mathrm{~dB}$, foram revoadas.

\section{Análise de Ruído da componenteTzz Free Air}
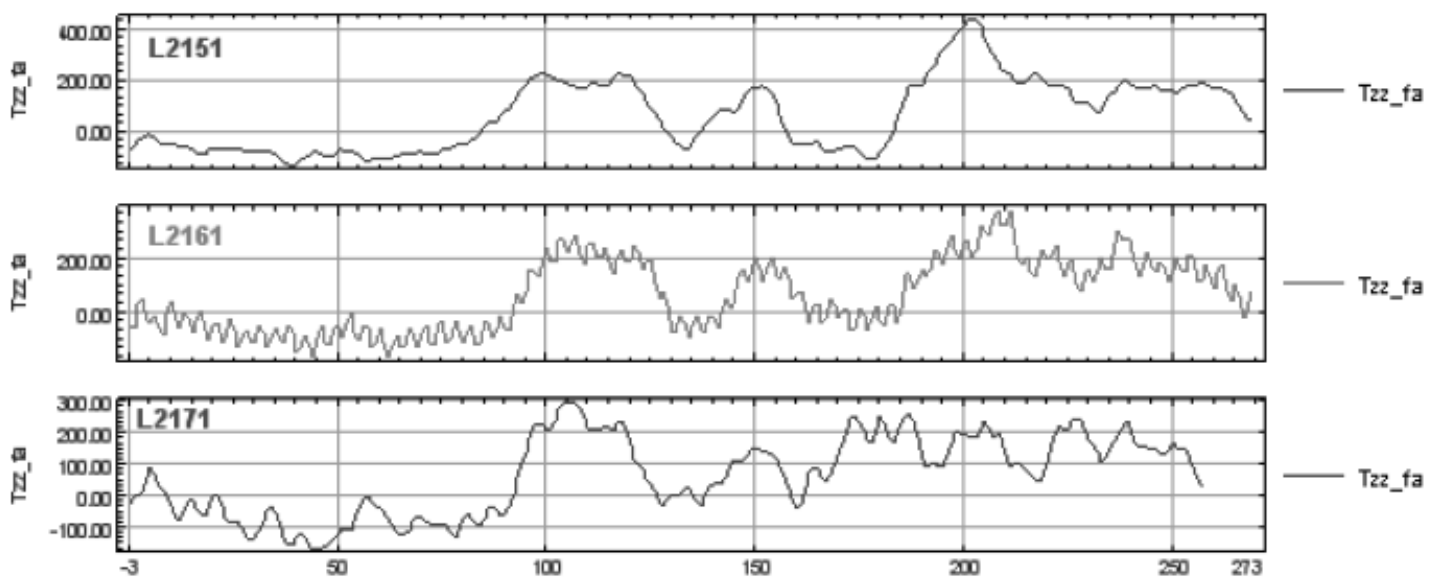

Figura 10 - Representação em perfil das componentes $T_{z z}$ Ar-Livre da área de Cebus 10. Note que a linha L2161 é altamente ruidosa, apresentando razão sinal ruído de aproximadamente $12 \mathrm{~dB}$ e que foi necessário ser revoada. Observe que a linha L2161, ruidosa, apresenta correspondência, provavelmente geológica, com as linhas L2151 e L2171.

\section{Componentes Free Air do Tensor X Componentes Sintéticas do Tensor}

Eo

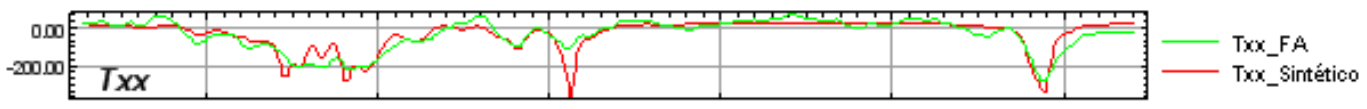

Eo

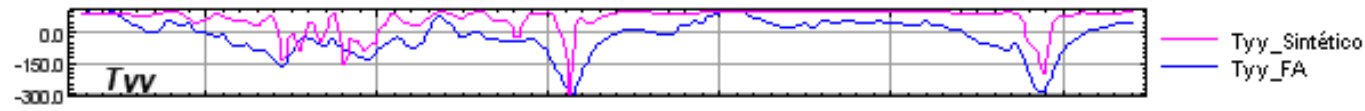

Eo

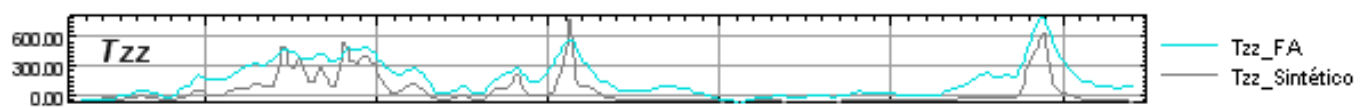

Eo

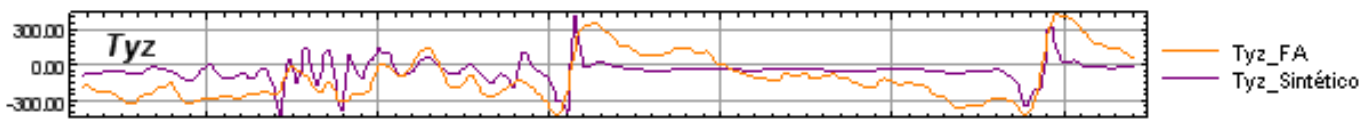

Eo

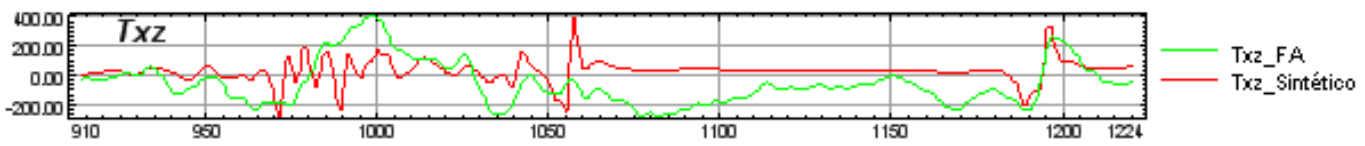

Figura 11 - Comparação entre cada uma das componentes do tensor de gradiente de gravidade dos dados medidos e calculados. Observe que existe uma grande similaridade entre as componentes medidas e as calculadas. 


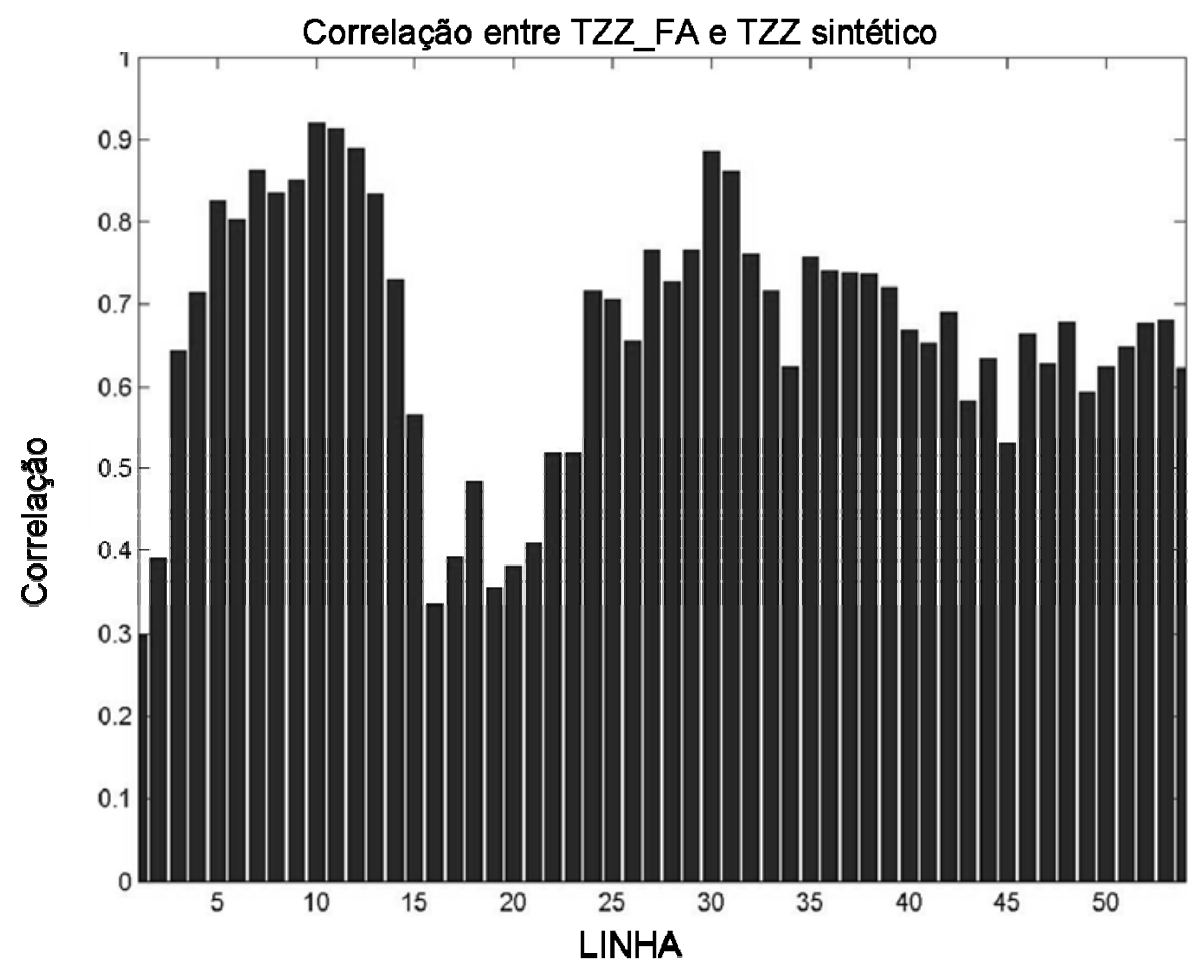

Figura 12 - Correlação entre as componentes $T_{z z}$ Free Air e as componentes $T_{z z}$ sintética adquiridas da aerogradiometria 3D-FTG da região do Baú-Gandarela.

\section{NOTAS SOBRE OS AUTORES}

Marco Antonio Braga. Doutor e Mestre em Geologia com ênfase em Geofísica Aplicada pela Universidade Federal do Rio de Janeiro/Colorado School of Mines em 2009. Especialista em métodos geofísicos aplicados à exploração de minério de ferro. Atualmente é coordenador de geofísica da área de Ferrosos da Vale.

Henry Galbiatti. Mestre em Geologia Estrutural e Mestre em Geotecnia pela Universidade Federal de Ouro Preto. Atualmente é gerente de Exploração Mineral de Ferrosos da Vale.

Dionísio Uendro Carlos. Bacharel e Mestre em Geofísica pelo IAG/USP, respectivamente em 2002 e 2006. Especialista em aerogamaespectrometria, tendo desenvolvido os primeiros blocos transportáveis de concreto radioativo para calibração de detectores gama portáteis e aerotransportados da América Latina. Atualmente trabalha na Vale, realizando controle de qualidade, processamento e interpretação de dados de 3D-FTG e aeromagnetometria para a exploração de minério de ferro.

Rodrigo Rocha Sousa. Técnico em Geologia e Mineração pelo CEFET-RN no ano de 2003. Tendo especialização em SIG, atualmente trabalha para a Vale na Gerência de Exploração Mineral de Ferrosos.

Tiago de Almeida. Mestrando em Geofísica Aplicada pela UFRGS. É especialista em métodos geofísicos aplicados ao minério de ferro. Trabalha na empresa FTGeo. 